ten MJ. Eradication of methicillin-resistant Staphylococcus aureus carriage: a systematic review. Clin Infect Dis 2009;48:922-930.

2. van Rijen MM, Bonten M, Wenzel RP, Kluytmans JA. Intranasal mupirocin for reduction of Staphylococcus aureus infections in surgical patients with nasal carriage: a systematic review. J Antimicrob Chemother 2008;61:254-261.

3. Kallen AJ, Wilson CT, Larson RJ. Perioperative intranasal mupirocin for the prevention of surgical-site infections: systematic review of the literature and meta-analysis. Infect Control Hosp Epidemiol 2005;26:916-922.

4. Robicsek A, Beaumont JL, Thomson RB Jr, Govindarajan G, Peterson LR. Topical therapy for methicillin-resistant Staphylococcus aureus colonization: impact on infection risk. Infect Control Hosp Epidemiol 2009;30:623-632.

5. Infectious Diseases Society of America, Emerging Infections Network. Perioperative Staphylococcus aureus Screening and Decolonization. Arlington, VA: Infectious Diseases Society of America, 2010.

6. Strom B, Kimmel S, eds. Textbook of Pharmacoepidemiology. Chichester: Wiley, 2006.

7. Hansen D, Patzke PI, Werfel U, Benner D, Brauksiepe A, Popp W. Success of MRSA eradication in hospital routine: depends on compliance. Infection 2007;35:260-264.

8. Caffrey AR, Quilliam BJ, LaPlante KL. Risk factors associated with mupirocin resistance in methicillin-resistant Staphylococcus aureus. J Hosp Infect 2010;76:206-210.

\section{Impact of PCR Testing for Clostridium difficile on Incident Rates and Potential on Public Reporting: Is the Playing Field Level?}

To the Editor-Healthcare-associated infections are a challenge to the healthcare field that pose an impact on the outcomes of patients. Preventive measures are implemented at most healthcare institutions to minimize the risks of acquiring healthcare-associated infections. ${ }^{1}$ Clostridium difficile is a fastidious anaerobic organism that is primarily responsible for antibiotic-associated colitis and accounts for about one-quarter of nosocomial antibiotic-associated diarrhea. ${ }^{2}$ In response to a recognized increase in disease activity and severity, the Ohio Department of Health made C. difficile infection (CDI) a reportable disease in 2006, ${ }^{3}$ and it was reinstated under the revised House Bill 197 in August 2010. While the benefits to transparency are numerous, the downside is that rates will be used for interhospital comparison, despite lack of adjustment for case mix. ${ }^{4}$ The intersection of public reporting for $\mathrm{CDI}$ in Ohio and the advent of testing based on polymerase chain reaction (PCR) adds another layer of complexity. On October 19, 2010, the Cleveland Clinic changed testing from enzyme immunoassay to a PCR test for $C$. difficile detection. We sought to compare our CDI rates before and after the institution of PCR-based testing and evaluate the effect this will have on our mandate for public reporting.
TABLE 1. Results of Testing of Consecutive Stool Samples for Clostridium difficile Using Enzyme Immunoassay (EIA) Toxin and Polymerase Chain Reaction (PCR) during a 3Month Period

\begin{tabular}{lccc}
\hline & EIA & PCR & $P$ \\
\hline No. of lab specimens & 2,579 & 2,534 & \\
Mean no. (\%) positive & $167(6.5)$ & $382(15.1)$ & $<.001$ \\
CDI rates $^{\mathrm{a}}$ & 4.9 & 10.3 & $<.001$ \\
\hline
\end{tabular}

NOTE. EIA toxin used before October 19, 2010, and PCR used after. CDI, Clostridium difficile infection.

a Cases per 10,000 patient-days.

CDI surveillance is performed prospectively at the Cleveland Clinic by infection preventionists. Cases are ascertained by daily review of lab reports of patients with positive stool tests for $C$. difficile, and chart review establishes the presence and onset of symptoms. Before October 19, 2010, testing was performed by enzyme immunoassay detection of toxins $A$ and $\mathrm{B}$ (Wampole), and after this date, the testing methodology was changed to PCR (BD Genehom). Query of the microbiology information systems was done to compare the results of testing for $\mathrm{CDI}$, using consecutive stool samples during a 3-month period before and after the change. A telephone survey of 11 other Ohio hospitals was conducted to determine whether PCR testing for CDI had been implemented at their institution.

The prevalence of positive tests for CDI increased significantly from $6.5 \%$ of 2,579 stools tested to $15.1 \%$ of 2,534 stools tested after introduction of PCR testing $(P<.001)$. The rate of CDI also increased significantly (from 4.9 per 10,000 patient-days to 10.3 ; Table 1 ). There was no identified $C$. difficile cluster after implementation of PCR testing to account for the increased percent positives observed. None of the 11 hospitals in Ohio contacted had introduced PCR testing for CDI during this time. There was a significant increase in the number and rates of CDI after the introduction of PCR testing that was unexplained by other reasons and not unexpected. Decisions about choice of diagnostic platform for CDI testing are complex and should not be driven by need for publicly reporting rates. However, public display of CDI rates are an implicit comparison of quality of care provided. In the case of CDI, identifying test methods should be included.

\section{ACKNOWLEDGMENTS}

Potential conflicts of interest. All authors report no conflicts of interest relevant to this article.

Kimberlee S. Fong, DO; ${ }^{1}$ Cynthia Fatica, RN, BSN; ${ }^{2}$ Geraldine Hall, PhD; ${ }^{3}$ Gary Procop, $\mathrm{MD}^{3}$

Susan Schindler, M(ASCP); $;^{3}$ Steven M. Gordon, MD; ${ }^{1}$ Thomas G. Fraser, MD

Affiliations: 1. Cleveland Clinic Department of Infectious Disease, Cleve- 
land, Ohio; 2. Cleveland Clinic Foundation, Infection Control, Cleveland, Ohio; 3. Cleveland Clinic Foundation, Laboratory, Cleveland, Ohio.

Address correspondence to Kimberlee S. Fong, DO, Cleveland Clinic Department of Infectious Disease, 9500 Euclid Avenue, Cleveland, $\mathrm{OH} 44195$ (fongk2@ccf.org).

Received May 16, 2011; accepted May 22, 2011; electronically published July $21,2011$.

Infect Control Hosp Epidemiol 2011;32(9):932-933

(C) 2011 by The Society for Healthcare Epidemiology of America. All rights reserved. 0899-823X/2011/3209-0020\$15.00. DOI: $10.1086 / 661789$

\section{REFERENCES}

1. Yokoe DS, Mermel LA, Anderson DJ, et al. A compendium of strategies to prevent healthcare-associated infections in acute care hospitals. Infect Control Hosp Epidemiol 2008;29(suppl 1): S12-S21.

2. Cohen $\mathrm{SH}$, Gerding DN, Johnson S, et al. Clinical practice guidelines for Clostridium difficile infection in adults: 2010 update by the Society for Healthcare Epidemiology of America (SHEA) and the Infectious Diseases Society of America (IDSA). Infect Control Hosp Epidemiol 2010;31:431-455.

3. Ohio Department of Health. Final report for rates of Clostridium difficile for Ohio hospitals and nursing homes January-December 2006. http://www.odh.ohio.gov/alerts/cdiffl.aspx.

4. Harris AD, McGregor JC. The importance of case-mix adjustment for infection rates and the need for more research. Infect Control Hosp Epidemiol 2008;29:693-694.

\section{Nosocomial Infections in Tbilisi, Georgia: A Retrospective Study of Microbiological Data from 4 Major Tertiary Care Hospitals}

To the Editor-Healthcare-associated infections (HAIs) are important and ever-increasing public health problems worldwide. These infections are associated with increased morbidity, length of stay, mortality, and costs. ${ }^{1-3}$ The problem is somewhat underrecognized in the country of Georgia. ${ }^{4}$ There are relatively scarce statistical data available regarding the epidemiology of nosocomial infections and the prevalence of infection due to multidrug-resistant (MDR) organisms in the region. During the past decade, several small-scale studies have tried to address the problem and clearly showed a significant burden of HAIs and high prevalence of MDR infections in Georgia. ${ }^{5-8}$

The goal of our study was to evaluate the epidemiology of nosocomial pathogens and their resistance patterns at 4 major tertiary care centers in Tbilisi, Georgia. A 3-year retrospective descriptive analysis of microbiological data collected during the period 2007-2010 from 4 major tertiary health care centers in Tbilisi, Georgia, was performed. All microbiology specimens were Gram stained and cultured at the same referral microbiology laboratory. Identification of the bacterial pathogens was performed with an automated system for identification and susceptibility tests (VITEK; bioMérieux). Antibiotic susceptibility testing was performed with the disk diffusion method or by using an automated method (VITEK; bioMérieux). Stool samples were assessed for Clostridium difficile with enzyme-linked immunosorbent assay for A and B toxin.

A total of 3,452 available clinical samples were included in the study, and positive findings were documented for 1,607 cultures $(46.6 \%)$. The most commonly isolated microorganisms included Klebsiella pneumonia (in 26.5\% of samples), Pseudomonas aeruginosa (15.2\%), Candida albicans (12.3\%), Staphylococcus aureus (9\%), Escherichia coli (7.6\%), and Acinetobacter baumannii (5.1\%). The susceptibility patterns of gram-negative rods (GNRs) to the most commonly used antibiotics are shown in Table 1. Among 95 GNR isolates tested for the presence of extended-spectrum $\beta$-lactamase (ESBL), $33.7 \%$ were found to be ESBL carriers. Extensive resistance to different groups of antibiotics was found among GNRs, including resistance to carbapenems. Only $29 \%$ and $11.9 \%$ of Pseudomonas and Acinetobacter isolates, respectively, were susceptible to imipenem. The vast majority of GNRs showed susceptibility to colistin, but we identified 8 colistin-resistant isolates, which included $P$. aeruginosa, K. pneumoniae, Proteus species, and E. coli. The most common gram-positive cocci (GPC) recovered were S. aureus, Staphylococcus epidermidis,

TABLE 1. Antibiotic Susceptibility of Selected Bacterial Pathogens

\begin{tabular}{lcccccccc}
\hline & \multicolumn{7}{c}{ Susceptibility to antibiotics, \% } \\
\cline { 2 - 9 } Bacterial pathogen & AMK & CEPH-3 & CFT & CFP & CIP & PIP/TAZ & IMP & FOSPH \\
\hline Klebsiella pneumoniae & 45.1 & 60.7 & 57.2 & 59.1 & 69.1 & 60.7 & 76.8 & 57.0 \\
Pseudomonas aeruginosa & 20.4 & 0 & 13.1 & 12.5 & 22.6 & 20.0 & 29.0 & 31.5 \\
Escherichia coli & 59.0 & 50.0 & 53.4 & 52.5 & 40.4 & 44.9 & 89.4 & 63.4 \\
Acinetobacter baumannii & 10.1 & 0 & 1.8 & 7.0 & 4.8 & 13.7 & 11.9 & 23.3 \\
Enterobacter aerogenes & 39.1 & 4.2 & 19.4 & 20.6 & 21.0 & 20.3 & 39.1 & 49.1 \\
Proteus mirabilis & 46.4 & 42.9 & 48.3 & 42.9 & 48.3 & 53.8 & 51.7 & 61.1 \\
Klebsiella oxytoca & 39.3 & 55.6 & 69.6 & 60.7 & 64.3 & 57.1 & 100.0 & 64.0 \\
Enterobacter cloacae & 54.5 & 42.9 & 36.4 & 42.9 & 59.1 & 55.0 & 71.4 & 66.7 \\
\hline
\end{tabular}

NOTE. AMK, amikacin; CEPH-3, third-generation cephalosporins other then ceftazidime; CFT, ceftazidime; CFP, cefepime; CIP, ciprofloxacin; FOSPH, fosphomycin; IMP, imipenem; PIP/TAZ, piperacillin-tazobactam. 